\title{
Timeliness of lung cancer care in Victoria: a retrospective cohort study
}

$\mathrm{M}$ ore Australian men and women die from lung cancer than from any other cancer. ${ }^{1}$ It is the fourth most common neoplasm in both men and women, and in 2014 more than 11000 people were diagnosed with lung cancer in Australia. ${ }^{1}$ The 5 -year survival rate is $14 \%{ }^{1}$ the median survival time for patients with non-small cell lung cancer (NSCLC) is 6.9 months, and for small cell lung cancer (SCLC) it is 7.2 months. $^{2}$

A recent Victorian study found that only $30 \%$ of patients with NSCLC received treatment with curative intent, and only $33 \%$ were discussed in a multidisciplinary team meeting. ${ }^{2}$ While $26 \%$ of patients presented with stage III disease, only $8 \%$ had invasive staging of the mediastinum, highlighting a potential discordance in staging of the cancer. The study did not assess delays in the care pathway.

In addition to obvious psychological distress, delay in managing lung cancer increases the potential for disease progression before treatment and may reduce the capacity for treatment with curative intent. ${ }^{3}$ Brocken and colleagues categorised delay as either "first-line", caused by delays in the patient seeking medical advice or a delay in management by the general practitioner, or "secondline", caused by delays in referral (time lag between the hospital receiving a referral and accessing a specialist) and treatment delivery (time lag between diagnosis and the start of treatment). ${ }^{4}$

Systematic reviews have identified organisational factors that affect the timeliness of care, including whether surgery was undertaken in a teaching hospital, whether the patient was initially referred to someone other than a respiratory physician, and the increasing number of diagnostic tests and hospitals attended to achieve a diagnosis. ${ }^{5} \quad$ Patient-level factors associated with second-line delays

\section{Abstract}

Objective: To assess factors associated with second-line delays in the management of patients diagnosed with lung cancer.

Design, setting and participants: A retrospective cohort study, conducted in six public and two private Victorian hospitals, of 1417 patients aged 18 years or more who were diagnosed between July 2011 and October 2014 with an incident case of lung cancer identified by International Classification of Diseases, 10th revision codes (C34.0-C34.9, Z85.1, Z85.2) on the basis of either a clinical or pathological diagnosis.

Outcome measures: Time intervals between referral, diagnosis and initial definitive management.

Results: The median time from referral to diagnosis was 15 days (interquartile range [IQR], 5-36); from diagnosis to initial definitive management, 30 days (IQR, 6-84); and from referral to initial definitive management, 53 days (IQR, 25-106). Factors that were significantly associated with delay between referral and initial definitive management include declining or not being referred to palliative care (hazard ratio [HR], $v$ patients referred for palliation, 0.73; 95\% Cl, 0.62-0.86; $P<0.001$ ), and being treated in a public hospital ( $\mathrm{HR}, v$ patients managed in a private hospital, 0.55; 95\% Cl, 0.48-0.64; $P<0.001$ ). The median time from referral to initial definitive management in public and private hospitals was 61 days (IQR, 35-118) and 30 days (IQR, 13-76) respectively; 48\% of patients in public hospitals waited longer than the British National Health Service target of a maximum 62 days between referral and first definitive treatment.

Conclusion: There are significant delays at various stages of the patient journey after referral for initial definitive management. Having a greater understanding of these delays will enable strategies to be developed that improve the timeliness of care for patients with lung cancer.

include presentation with atypical symptoms, ${ }^{6}$ fewer years of education, lower disposable income, and multiple comorbidities, ${ }^{7}$ as well as symptoms that suggest less advanced disease. ${ }^{8}$ The reported impact of the age of the patient on timeliness of care is variable. ${ }^{5}$

In this article we examine second-line delays in the management of NSCLC in Victorian hospitals.

\section{Materials and methods}

\section{Patients}

Data were sourced from the Victorian Lung Cancer Registry (VLCR). This initiative operates in eight Victorian hospitals (six public hospitals, including four metropolitan and two regional centres, and two private hospitals) and captures about $25 \%$ of Victorian lung cancer notifications. ${ }^{9}$
Patients were recruited to the registry if they were at least 18 years old and presented with an incident case of lung cancer identified by ICD-10 (International Classification of Diseases, 10th revision) lung cancer codes (C34.0-C34.9, Z85.1, Z85.2) based on either a clinical or pathology diagnosis. Patients were excluded if they had secondary lung cancer or mesothelioma.

Patients diagnosed with NSCLC between July 2011 and October 2014 were assessed for eligibility. A waiver of consent enabled details about deceased patients to be collected. Of the eligible 1863 patients, 446 (24\%) were excluded: 267 $(14.3 \%)$ had been diagnosed by doctors who had not consented to participation in the registry, 67 (3.6\%) had a carcinoid tumour, $14(0.7 \%)$ had mesothelioma, and 98 (5.3\%) declined participation. 


\section{Data collection}

Hospital lung cancer notifications were provided by participating hospitals each month. Medical record review was undertaken 4 months after diagnosis. Confirmation of management was obtained when registry staff contacted patients by telephone 6 and 12 months after diagnosis. The medical records of patients who died after diagnosis but before follow-up were used as the only source of information for these patients.

\section{Statistical considerations}

Categorical data are presented as absolute numbers and percentages. Continuous variables are presented as means and standard deviations for normally distributed data and medians and interquartile ranges (IQR) for non-parametric data. Time intervals were recorded as medians, IQRs and means.

Patient factors that were analysed included sex, age, country of birth, preferred language, smoking status, TNM stage of disease at diagnosis, ${ }^{10}$ Eastern Cooperative Oncology Group (ECOG) performance status, ${ }^{11}$ and major comorbidities extracted from their medical records. Age groups were categorised by quartiles. Patients with diabetes mellitus were defined as those with insulindependent or oral hypoglycaemic disease; patients with renal disease were defined as those requiring dialysis; patients with cardiovascular disease were defined as those with a previous myocardial infarction or coronary intervention; patients with respiratory disease were defined as those with a functional expiratory volume of less than $66 \%$; and patients with neoplastic disease were defined as those with any past history of cancer other than lung cancer. The Colinet simplified comorbidity score (SCS) is a weighted index with a range of 0 (no comorbidities) to $20 .^{12}$ The index was dichotomised into two categories $(>9 v \leq 9)$, in line with evidence that an SCS greater than 9 predicts worse survival for patients with NSCLC. ${ }^{13}$ The Index of Relative Socio-economic Advantage and Disadvantage (IRSAD), which rates the socio-economic status of the patient on the basis of their residential postcode, was categorised into deciles; a low score indicates relatively greater disadvantage and a lack of advantage in general. ${ }^{14}$

Disease management factors included in our analysis included whether the patient received surgery, radiotherapy or chemotherapy as their initial treatment, and whether the intent was curative or palliative. Organisational factors included hospital type (private $\mathrm{v}$ public) and location (metropolitan $\mathrm{v}$ regional) for the hospital where diagnosis and initial definitive management were provided.

Three main outcomes were investigated:

- the interval between initial referral for management and diagnosis ("referral to diagnosis");

- the interval between diagnosis and initial surgery, chemotherapy, radiotherapy or referral to palliative care ("diagnosis to initial definitive management"); and

- the interval between referral and initial definitive management.

The referral date was the date recorded on the referral letter to the hospital or diagnosing clinician. Survival was censored at the date of the event of interest or the date on which the patient died; otherwise, the last known follow-up date (30 December 2014) was used. The log-rank test was used to compare survival in subgroups.

Both univariate and separate multivariate Cox regression analyses were performed to identify independent and significant factors associated with each time interval. For the multivariate model, we started with all significant variables identified in the univariate analysis and applied the stepwise method to determine the final list of variables included in the multivariate model. The likelihood ratio test was performed, with the probability of entry and removal of the variables set at 0.01 and 0.05 respectively. The median time to event was computed from the survival curve (along with the IQR). The proportional hazards assumption was tested using the Schoenfeld test. Data analysis was performed in Stata 13.0 (StataCorp).

\section{Ethics approval}

Ethics approval was provided by the Monash University Human Research Ethics Committee (reference CF11/ 1693-2011000940).

\section{Results}

The demographic and clinical characteristics of the 1417 patients included in our analysis are summarised in Box 1. The mean age of the cohort was $71.3 \pm 11.4$ years. The mean SCS was $7.6 \pm 2.9$. There were 682 deaths in the cohort (48\%).

The median interval between referral and diagnosis was 15 days (IQR, 5-36 days), between diagnosis and initial definitive management 30 days (IQR, 6-84 days), and between referral and definitive management 53 days (IQR, 25-106 days) (Box 2). Socio-demographic factors associated with the length of the intervals between referral and diagnosis, diagnosis and initial definitive management, and referral and initial definitive management are summarised in Box 2. Box 3 includes the clinical factors associated with more timely diagnosis after referral. Hospital-related factors are summarised in Box 4.

Box 5 summarises the results of the stepwise selection process, and details the factors associated with overall delay for each of the three time intervals. The proportional hazards assumption was not violated in the final multivariate model, except for the interval between diagnosis and first management $(P<0.001)$. This violation was largely associated with the wider differences in the survival slope during the early periods of follow-up for the surgery and no-surgery groups, but, as the plots did not cross at any time point, we decided to retain this variable in the model because it was deemed a clinically important factor.

A longer interval between referral and diagnosis was associated with being born overseas; having early 
1 Demographic and clinical characteristics of the 1417 patients

\section{Characteristics}

Age (mean, $71.3 \pm 11.4$ years)

Sex

Born overseas

Preference for language other than English

Ever smoked

Notifying hospital type

Categories

$\leq 64$ years

65-72 years

$73-80$ years

$\geq 81$ years

Male

Number of patients (percentage)

$369(26.0 \%)$

$370(26.1 \%)$

$358(25.3 \%)$

$320(22.6 \%)$

$832(58.7 \%)$

719 (50.7\%)

$86(6.1 \%)$

1139 (90\%)

$1215(85.7 \%)$

$202(14.3 \%)$

$428(30.2 \%)$

$989(69.8 \%)$

Comorbidities (ascertained from medical record review)

SCS (mean, $7.6 \pm 2.9$ )

ECOG score

Disease stage at diagnosis* Regional

Private

Public

Diabetes mellitus

Renal disease

Cardiovascular disease

Respiratory disease

Neoplastic disease

$>9$

$<2$

2-4

Not available/not stated

195 (13.8\%)

$24(1.7 \%)$

$244(17.2 \%)$

$206(14.5 \%)$

$292(20.6 \%)$

$550(38.8 \%)$

$528(37.3 \%)$

$194(13.7 \%)$

695 (49.1\%)

$122(8.6 \%)$

$135(9.5 \%)$

$201(14.2 \%)$

$253(17.9 \%)$

$706(49.8 \%)$
IRSAD, percentiles (mean score: $1020[S D, 73])$

$1 \%-20 \%$

$21 \%-40 \%$

$41 \%-60 \%$

$61 \%-80 \%$

$81 \%-100 \%$

First treatment intent was

curative

Surgery performed

Chemotherapy performed

Radiotherapy performed

Palliative care

Referral

Not performed/declined

Not stated

Treating hospital for surgery

Metropolitan

Regional

Private

Public

Metropolitan

Regional

Private

Public

Metropolitan

Regional

Private

Public

$206(14.6 \%)$

$184(13.0 \%)$

$246(17.4 \%)$

$276(19.5 \%)$

$502(35.5 \%)$

$461(44.2 \%)$

459 (32.4\%)

548 (38.7\%)

487 (34.4\%)

404 (28.5\%)

835 (58.9\%)

178 (12.6\%)

437 (95.2\%)

$22(4.8 \%)$

179 (39.0\%)

280 (61.0\%)

406 (75.9\%)

$129(24.1 \%)$

$177(33.1 \%)$

358 (66.9\%)

367 (77.4\%)

$107(22.6 \%)$

$71(15.0 \%)$

403 (85.0\%)

ECOG = Eastern Cooperative Oncology Group ${ }^{11} ;$ IRSAD = Index of Relative Socio-economic

Advantage and Disadvantage ${ }^{14} ; \mathrm{SCS}=$ Colinet simplified comorbidity score $(\mathrm{SCS})^{12} ; \mathrm{SD}=$ standard $^{2}$

deviation. * TNM classification of malignant tumours (7th edition).$^{10}$ stage disease (stage I or II) or not having the stage documented; notification by a public hospital; receiving curative treatment; and either declining or not receiving palliative care. Patients waited significantly longer for initial definitive management after diagnosis if their ECOG performance status was not documented; having stage II or III disease, or the disease stage at diagnosis was not documented; receiving subsequent treatment in a public hospital; and not undergoing surgery. Overall, a longer interval between referral and initial definitive management was associated with being managed in a public hospital and not receiving (or declining) palliative care.

\section{Discussion}

Using a clinical quality registry, we reviewed care provided to a large cohort of patients with NSCLC who were managed in Victorian hospitals. We found several disparities. Patients born overseas experienced delays in receiving a diagnosis after referral, but not in treatment after diagnosis; overall, their treatment path took longer than that of Australian-born patients. Patients with advanced disease received prompt diagnosis, but then experienced delays in further definitive management. This may be explained by patients only needing palliative care after the diagnosis was confirmed. Conversely, patients with early stage disease waited longer to receive a diagnosis but, once diagnosed, received more timely initial definitive management than patients with more advanced disease. Patients managed in public hospitals waited longer than patients managed in the private sector to receive either a diagnosis or initial definitive management. Patients receiving chemotherapy in regional hospitals waited longer than those in metropolitan hospitals. Any active treatment took longer to commence than palliation.

The association between timeliness of care delivery and health outcomes 
2 Socio-demographic factors associated with length of the time intervals (in days) between referral and diagnosis, diagnosis and first management, and referral and first definitive management

\begin{tabular}{|c|c|c|c|c|c|c|}
\hline \multirow[b]{2}{*}{ Characteristic } & \multicolumn{2}{|c|}{ Referral to diagnosis } & \multicolumn{2}{|c|}{$\begin{array}{l}\text { Diagnosis to initial } \\
\text { definitive management }\end{array}$} & \multicolumn{2}{|c|}{$\begin{array}{c}\text { Referral to initial } \\
\text { definitive management }\end{array}$} \\
\hline & Median (IQR) & P* & Median (IQR) & P* & Median (IQR) & P* \\
\hline Overall & $15(5-36)$ & & $30(6-84)$ & & $53(25-106)$ & \\
\hline Age group & & 0.133 & & $<0.001$ & & $<0.001$ \\
\hline$\leq 64$ years & $14(5-32)$ & & $24(5-60)$ & & $43(20-81)$ & \\
\hline $65-72$ years & $16(5-41)$ & & $25(0-66)$ & & $47(23-100)$ & \\
\hline $73-80$ years & $16(6-40)$ & & $31(3-75)$ & & $56(32-112)$ & \\
\hline$\geq 81$ years & $13(4-31)$ & & $46(14-N C)$ & & $68(31-421)$ & \\
\hline \multicolumn{7}{|l|}{ Sex } \\
\hline Female & $13(5-34)$ & 0.498 & $31(5-97)$ & 0.271 & $52(24-120)$ & 0.282 \\
\hline Male & $16(5-37)$ & & $30(7-75)$ & & $53(25-102)$ & \\
\hline \multicolumn{7}{|l|}{ Place of birth } \\
\hline Australia & $13(4-33)$ & 0.001 & $26(1-82)$ & 0.063 & $46(20-102)$ & 0.019 \\
\hline Overseas & $18(6-40)$ & & $33(10-88)$ & & $57(31-112)$ & \\
\hline \multicolumn{7}{|c|}{ Language preference } \\
\hline English & $15(5-35)$ & 0.031 & $30(6-84)$ & 0.491 & $51(23-105)$ & 0.031 \\
\hline Other language & $20(7-61)$ & & $36(14-72)$ & & $70(42-131)$ & \\
\hline \multicolumn{7}{|l|}{ Smoking history } \\
\hline Never smoked & $12(4-31)$ & 0.398 & $27(1-59)$ & 0.456 & $43(17-87)$ & 0.175 \\
\hline Ever smoked & $16(6-38)$ & & $28(4-70)$ & & $53(26-105)$ & \\
\hline IRSAD deciles & & 0.533 & & 0.089 & & 0.068 \\
\hline 1 & $19(7-40)$ & & $34(7-80)$ & & $56(29-105)$ & \\
\hline 2 & $27(11-44)$ & & $50(4-434)$ & & $73(34-261)$ & \\
\hline 3 & $15(8-34)$ & & $26(0-63)$ & & $52(3-96)$ & \\
\hline 4 & $14(6-40)$ & & $51(7-731)$ & & $62(33-147)$ & \\
\hline 5 & $17(6-45)$ & & $29(1-88)$ & & $61(20-111)$ & \\
\hline 6 & $14(5-35)$ & & $28(3-88)$ & & $43(24-100)$ & \\
\hline 7 & $16(7-32)$ & & $29(4-69)$ & & $59(26-98)$ & \\
\hline 8 & $13(4-37)$ & & $28(6-59)$ & & $43(21-89)$ & \\
\hline 9 & $13(3-39)$ & & $29(9-90)$ & & $59(26-118)$ & \\
\hline 10 & $11(3-25)$ & & $25(8-63)$ & & $43(19-94)$ & \\
\hline
\end{tabular}

IRSAD = Index of Relative Socio-economic Advantage and Disadvantage; NC = not computable. * Log-rank test comparison across each category for the respective variable.

has been investigated in many studies, often with paradoxical results. ${ }^{15}$ This is partly explained by evidence that patients with more advanced disease are often fasttracked for referral and definitive management, but are less likely to survive than patients with less advanced disease. Of concern is the accumulating evidence that delay in treatment allows time for tumour growth and may result in patients becoming ineligible for curative treatment. Upstaging rates of $17 \%$ within a period of 20 days $^{16}$ and gross tumour volume increases of $35 \%$ over a median time of 13 days ${ }^{17}$ have been reported.

A systematic review published in 2009 reported that all eight studies which had examined time from referral to first respiratory specialist visit had found median waiting times of no more than 14 days. ${ }^{5}$ The only published Australian study identified a median time between referral and initial consultation of 5 days, and a further 14 days between initial consultation and a management decision. ${ }^{18}$ In contrast, median waiting times in our study were as high as 34 days for patients with early stage disease, and patients referred to a metropolitan hospital in which they subsequently underwent surgery waited a median 29 days after referral for a diagnosis.

The time between diagnosis and initiation of treatment for patients with lung cancer has been studied in 
3 Clinical factors associated with length of time intervals (in days) between referral to diagnosis, diagnosis to first management, and referral to first definitive management

\begin{tabular}{|c|c|c|c|c|c|c|}
\hline \multirow[b]{2}{*}{ Characteristic } & \multicolumn{2}{|c|}{ Referral and diagnosis } & \multicolumn{2}{|c|}{$\begin{array}{l}\text { Diagnosis and initial } \\
\text { definitive management }\end{array}$} & \multicolumn{2}{|c|}{$\begin{array}{c}\text { Referral and initial } \\
\text { definitive management }\end{array}$} \\
\hline & Median (IQR) & $P^{*}$ & Median (IQR) & $P^{*}$ & Median (IQR) & $P^{*}$ \\
\hline \multicolumn{7}{|l|}{ Comorbidities $^{\dagger}$} \\
\hline Diabetes mellitus present & $18(5-44)$ & 0.395 & $29(3-58)$ & 0.048 & $55(31-91)$ & 0.635 \\
\hline Diabetes mellitus absent & $14(5-35)$ & & $30(6-90)$ & & $52(24-108)$ & \\
\hline Renal disease present & $15(2-40)$ & 0.746 & $31(19-49)$ & 0.876 & $49(32-182)$ & 0.679 \\
\hline Renal disease absent & $15(5-36)$ & & $30(6-84)$ & & $53(24-106)$ & \\
\hline Cardiovascular disease present & $20(6-50)$ & 0.024 & $25(4-59)$ & 0.023 & $55(27-106)$ & 0.910 \\
\hline Cardiovascular disease absent & $14(5-34)$ & & $31(6-88)$ & & $52(24-106)$ & \\
\hline Respiratory disease present & $25(10-51)$ & $<0.001$ & $40(12-85)$ & 0.121 & $69(40-129)$ & $<0.001$ \\
\hline Respiratory disease absent & $14(4-33)$ & & $28(6-84)$ & & $50(22-102)$ & \\
\hline Neoplastic disease present & $16(5-40)$ & 0.156 & $30(4-77)$ & 0.516 & $55(24-128)$ & 0.653 \\
\hline Neoplastic disease absent & $15(5-36)$ & & $30(6-85)$ & & $52(25-104)$ & \\
\hline $\operatorname{SCS}<9$ & $15(6-36)$ & 0.345 & $30(4-77)$ & 0.281 & $54(26-111)$ & 0.191 \\
\hline $\mathrm{SCS} \geq 9$ & $15(4-36)$ & & $30(8-94)$ & & $50(22-102)$ & \\
\hline ECOG score $<2$ & $14(5-34)$ & $<0.001$ & $24(1-51)$ & $<0.001$ & $47(22-87)$ & $<0.001$ \\
\hline ECOG score $2-4$ & $9(3-22)$ & & $40(12-140)$ & & $56(24-244)$ & \\
\hline ECOG score missing & $18(6-44)$ & & $37(2-218)$ & & $59(29-129)$ & \\
\hline Disease stage at diagnosis ${ }^{\ddagger}$ & & $<0.001$ & & $<0.001$ & & 0.078 \\
\hline I & $34(16-61)$ & & $0(0-37)$ & & $56(33-103)$ & \\
\hline II & $29(11-60)$ & & $28(0-58)$ & & $59(33-102)$ & \\
\hline III & $17(7-28)$ & & $38(15-64)$ & & $55(34-83)$ & \\
\hline IV & $10(4-23)$ & & $30(12-72)$ & & $44(22-91)$ & \\
\hline Not available/not stated & $13(4-34)$ & & $32(7-339)$ & & $54(20-161)$ & \\
\hline First treatment intent non-curative & $12(4-24)$ & $<0.001$ & $11(25-47)$ & $<0.001$ & $37(19-63)$ & 0.017 \\
\hline First treatment intent curative & $28(11-58)$ & & $0(0-31)$ & & $45(22-78)$ & \\
\hline Surgery performed & $28(10-56)$ & $<0.001$ & $0(0-22)$ & $<0.001$ & $42(18-73)$ & $<0.001$ \\
\hline Surgery not performed & $11(4-25)$ & & $48(21-393)$ & & $61(30-230)$ & \\
\hline Chemotherapy performed & $13(4-30)$ & $<0.001$ & $24(7-47)$ & $<0.001$ & $38(19-68)$ & $<0.001$ \\
\hline Chemotherapy not performed & $16(6-42)$ & & $41(4-\mathrm{NC})$ & & $69(32-414)$ & \\
\hline Radiotherapy performed & $13(4-25)$ & $<0.001$ & $28(11-52)$ & $<0.001$ & $42(21-73)$ & $<0.001$ \\
\hline Radiotherapy not performed & $17(5-43)$ & & $32(0-739)$ & & $63(30-181)$ & \\
\hline Palliative care referral & $11(3-22)$ & $<0.001$ & $43(19-255)$ & $<0.001$ & $56(26-175)$ & 0.007 \\
\hline Palliative care not performed & $19(7-46)$ & & $23(0-55)$ & & $51(27-97)$ & \\
\hline Not stated & $8(2-24)$ & & $54(10-N C)$ & & $44(17-N C)$ & \\
\hline
\end{tabular}

ECOG = Eastern Cooperative Oncology group ${ }^{11} ;$ IRSAD = Index of Relative Socio-economic Advantage and Disadvantage ${ }^{14} ; \mathrm{NC}=$ not computable; SCS $=$ Colinet simplified comorbidity score (SCS). ${ }^{12} *$ Log-rank test comparison across each category for the respective variable. The difference associated with ECOG scores $(<2 \vee 2-4)$ was also significant if the not available/not stated group was excluded $(P<0.001)$. The comparisons for comorbidities are between those who had the comorbidity versus those who did not. + Comorbidities ascertained from medical record review. $\ddagger$ TNM classification of malignant tumours (7th edition)..$^{10}$

a number of countries, and the median was found to range between 12 and 52 days. ${ }^{5}$ Large cohort studies in Canada have identified waiting times of 42 days for curative radiotherapy and 39 days for surgery.
Closer to home, the median overall waiting time from diagnosis to initiation of radiation therapy in Queensland was 33 days; this was not affected by distance to the treating hospital. ${ }^{19}$ Median waiting times were longer for patients with early stage disease (stage I and II NSCLC; median, 48 days) than for those with more advanced disease (stages III and IV disease; median, 34 and 26 days respectively). Our finding 
4 Hospital-related factors associated with length of time intervals (in days) between referral and diagnosis, diagnosis and first management, and referral and first definitive management

\begin{tabular}{|c|c|c|c|c|c|c|}
\hline \multirow[b]{2}{*}{ Characteristic } & \multicolumn{2}{|c|}{ Referral to diagnosis } & \multicolumn{2}{|c|}{$\begin{array}{l}\text { Diagnosis to initial } \\
\text { definitive management }\end{array}$} & \multicolumn{2}{|c|}{$\begin{array}{c}\text { Referral to initial } \\
\text { definitive management }\end{array}$} \\
\hline & Median (IQR) & $P^{*}$ & Median (IQR) & $P^{*}$ & Median (IQR) & $P^{*}$ \\
\hline \multicolumn{7}{|l|}{ Notifying hospital } \\
\hline Metropolitan hospital & $15(5-38)$ & 0.289 & NA & & NA & \\
\hline Regional hospital & $18(10-29)$ & & NA & & NA & \\
\hline Private hospital & $7(2-19)$ & $<0.001$ & NA & & NA & \\
\hline Public hospital & $19(8-43)$ & & NA & & NA & \\
\hline \multicolumn{7}{|l|}{ Treating hospital } \\
\hline Private & NA & & $15(1-52)$ & & $30(13-76)$ & \\
\hline Public & NA & & 36 (11-91) & $<0.001$ & $61(35-118)$ & $<0.001$ \\
\hline \multicolumn{7}{|c|}{ Treating hospital for surgery } \\
\hline Metropolitan & $29(9-59)$ & 0.250 & $0(0-20)$ & 0.065 & $41(18-73)$ & 0.741 \\
\hline Regional & $23(16-44)$ & & $21(0-58)$ & & $44(34-72)$ & \\
\hline Private & $15(5-38)$ & $<0.001$ & $0(0-16)$ & 0.040 & $22(10-46)$ & $<0.001$ \\
\hline Public & $35(15-70)$ & & $0(0-28)$ & & $51(30-91)$ & \\
\hline \multicolumn{7}{|c|}{ Treating hospital for chemotherapy } \\
\hline Metropolitan & $11(3-90)$ & 0.010 & $20(6-40)$ & 0.001 & $37(17-65)$ & 0.068 \\
\hline Regional & $20(10-31)$ & & $32(11-58)$ & & $43(28-79)$ & \\
\hline Private & $5(1-16)$ & $<0.001$ & $14(4-30)$ & $<0.001$ & $20(11-38)$ & $<0.001$ \\
\hline Public & $19(8-37)$ & & $29(9-51)$ & & $50(30-78)$ & \\
\hline \multicolumn{7}{|c|}{ Treating hospital for radiotherapy } \\
\hline Metropolitan & $12(4-25)$ & 0.964 & $26(10-49)$ & 0.099 & $41(20-70)$ & 0.515 \\
\hline Regional & $16(6-26)$ & & $35(15-61)$ & & $50(23-78)$ & \\
\hline Private & $3(1-8)$ & $<0.001$ & $12(6-31)$ & 0.005 & $20(12-43)$ & $<0.001$ \\
\hline Public & $15(7-29)$ & & $30(13-54)$ & & $46(25-76)$ & \\
\hline
\end{tabular}

$\mathrm{NA}=$ not available. $*$ Log-rank test comparison across each category for the respective variable. $\bullet$

that the median waiting time from diagnosis to initiation of radiotherapy in both private and public hospitals was 28 days is comparable with these Queensland findings. However, when our analysis was confined to waiting times in public hospitals, the median waiting time was 30 days, compared with 12 days for patients treated in private facilities.

Dutch quality indicators suggest that organisations should provide a diagnosis within 21 days, and commence treatment within 35 days of the first visit to a specialist. ${ }^{20}$ The Danish Lung Cancer Registry has established targets of 28 days for the period from referral to diagnosis, 14 days from diagnosis to initial treatment, and 42 days from referral to initial treatment. ${ }^{21}$ Current standards set by the British National Health Service (NHS) require that all patients with suspected cancer be seen by a specialist within 14 days, and that those diagnosed with cancer be treated within 31 days of the decision to treat and within 62 days of referral. $^{22}$ While setting a generic waiting time for all cancer management has been criticised as not considering the different levels of priority for the treatment of the various cancer types, ${ }^{23}$ such targets acknowledge the psychological impact of delay on patients, regardless of the prognosis of the cancer. A study conducted in two Australian radiotherapy centres found that delay between a decision to give radiotherapy and starting treatment (ie, between diagnosis and treatment) caused a higher level of concern than a delay between referral and diagnosis. ${ }^{24}$

Our finding that patients with less advanced disease waited longer for a diagnosis than patients with advanced disease is consistent with a United Kingdom study of general practitioner referral patterns which showed that a greater proportion of urgent than of non-urgent referrals involved patients with advanced lung cancer disease (higher TNM stage, and extensive $\mathrm{V}$ limited stage) ${ }^{25}$ However, we found that the interval between diagnosis and treatment was shorter for patients with early stage disease (stage I), suggesting that they were given priority for treatment over patients with stage II or III disease. 
5 Multivariate analysis of factors associated with lengths of intervals Characteristics Hazard ratio $(95 \% \mathrm{Cl}) *$

Characteristics affecting time from referral to diagnosis

Place of birth

\section{Australia \\ Overseas} 1

$0.84(0.72-0.99)$

0.035

Disease stage at diagnosis ${ }^{\ddagger}$

$$
\text { I }
$$

$0.58(0.43-0.78)$

0.000

II

III

IV

Not available/not stated

Notifying hospital

Private

0.66 (0.49-0.89)

$0.92(0.72-1.18)$

1

$0.74(0.59-0.93)$

0.010

Public

1

$0.50(0.41-0.60)$

First treatment intent

Non-curative

1

Curative

$0.73(0.61-0.89)$

0.002

Palliative Care

Yes

No/declined

1

Not stated

0.006

0.529
$<0.001$

0.245

$1.22(0.87-1.71)$
Factors affecting time from diagnosis to initial definitive management ECOG performance status

$<2$

1

$2-4$

$0.95(0.77-1.16)$

$0.84(0.74-0.96)$

Disease stage at diagnosis ${ }^{\dagger}$

$$
\begin{aligned}
& \text { I } \\
& \text { II } \\
& \text { III } \\
& \text { IV }
\end{aligned}
$$

Not available/not stated

Treating hospital type

Private

Public

Surgery performed

Yes

No/declined

Factors affecting time from referral to initial definitive management

Palliative care

Yes

No/declined

1

Not stated

$0.73(0.62-0.86)$

$1.03(0.78-1.35)$

Treating hospital type

Private

1

Public

$0.55(0.48-0.64)$
Our finding that overseas-born patients waited, on average, 5 days longer than their Australian-born counterparts for a diagnosis after referral may reflect organisational (eg, access to translators and services) or patient-level barriers (eg, cultural and health literacy). Cultural barriers explained a $30 \%$ reduction in African Americans receiving cancer stage-appropriate treatment in four United States hospitals; African Americans were more likely than their white counterparts to report fatalism, negative surgical beliefs and mistrust of doctors. ${ }^{26}$ Further work is required to unravel the reasons for the delays experienced by overseasborn patients presenting with suspected lung cancer in Australia.

The finding that patients managed in public hospitals waited more than twice as long as those treated in private hospitals for diagnosis and treatment is disappointing, Particularly unsatisfactory was that the median time from referral to initiation of definitive treatment was 61 days in public hospitals, only just within the British NHS target of a maximum 62 days between referral and first definitive treatment of cancer patients. ${ }^{22}$ The overall proportion of patients who waited longer than 62 days in our study was $42 \%$, but for patients treated in public hospitals it was $48 \%$.

There were several limitations to our study. First, $20 \%$ of patients were excluded from analysis because either the patient or their treating doctor declined participation in the registry. We cannot ascertain whether there were systematic differences in disease management patterns of these patients. Second, caution should be exercised when extrapolating our findings to Victorian hospitals that do not contribute to the VLCR. Patients notified from regional hospitals are underrepresented in the registry $(15 \%$ of cases in our registry were notified from regional Victorian hospitals, while $33 \%$ of lung cancer notifications in Victoria over the past 5 years were by regional hospitals ${ }^{27}$ ). Finally, the IRSAD score provides socioeconomic characteristics of areas in which patients lived at the time of

ECOG $=$ Eastern Cooperative Oncology group. *A positive hazard ratio corresponds to shorter time to event. † Cox proportional hazards model. $¥$ TNM classification of malignant tumours (7th edition).$^{10}$ 
their diagnosis; we were unable to investigate the impact of an individual's socio-economic status on the timeliness of care.

Improving timeliness of care requires political will and investment in resources to redesign care processes. Interventions that improve timeliness and appropriateness of care, including rapid access lung cancer clinics $^{28}$ and process restructuring that enables rapid diagnostic imaging, biopsy collection and progression to discussion at multidisciplinary team meetings ${ }^{29}$ have produced impressive results, and warrant investigation in Victorian public hospitals. Any intervention should be underpinned by system- atic monitoring of its impact on the quality of care and feedback to clinical units, such as that provided by the VLCR.

Competing interests: No relevant disclosures.

Received 9 Sep 2015, accepted 20 Nov 2015.

(c) 2016 AMPCo Pty Ltd. Produced with Elsevier B.V. All rights reserved. 
1 Australian Institute of Health and Welfare. Cancer in Australia: an overview 2014. Canberra: AlHW, 2010. (Cat. No. CAN 88; Cancer series, number 90.) http://www.aihw.gov.au/ WorkArea/DownloadAsset.aspx?id= 60129550202 (accessed Nov 2015).

2 Mitchell PL, Thursfield VJ, Ball DL, et al. Lung cancer in Victoria: are we making progress? Med J Aust 2013; 199: 674-679. https://www.mja.com.au/ journal/2013/199/10/lung-cancervictoria-are-we-making-progress

3 Everitt S, Herschetal A, Callahan J, et al. High rates of tumor growth and disease progression detected on serial pretreatment fluorodeoxyglucosepositron emission tomography/ computed tomography scans in radical radiotherapy candidates with nonsmall cell lung cancer. Cancer 2010; 116: 5030-5037.

4 Brocken P, Kiers BA, Looijen-Salamon MG, et al. Timeliness of lung cancer diagnosis and treatment in a rapid outpatient diagnostic program with combined ${ }^{18}$ FDG-PET and contrast enhanced CT scanning. Lung Cancer 2012; 75: 336-341.

5 Olsson JK, Schultz EM, Gould MK. Timeliness of care in patients with lung cancer: a systematic review. Thorax 2009; 64: 749-756.

6 Bjerager M, Palshof T, Dahl R, et al. Delay in diagnosis of lung cancer in general practice. Br J Gen Pract 2006; 56: 863-868.

7 Dalton SO, Frederiksen BL, Jacobsen E, et al. Socioeconomic position, stage of lung cancer and time between referral and diagnosis in Denmark, 2001-2008. Br J Cancer 2011; 105: 1042-1048.

8 Powell AA, Schultz EM, Ordin DL, et al. Timeliness across the continuum of care in veterans with lung cancer. J Thor Oncol 2008; 3: 951-957.

9 Stirling R, Evans S, McLaughlin P, et al. The Victorian Lung Cancer Registry pilot: improving the quality of lung cancer care through the use of a disease quality registry. Lung 2014; 192: 749-758.

10 Sobin LH, Gospodarowicz MK, Wittekind C, editors; International Union Against Cancer. TNM classification of malignant tumours, 7th edition. Hoboken, NJ: Wiley-Blackwell, 2009.

11 Oken MM, Creech, RH, Tormey, DC, et al. Toxicity and response criteria of the Eastern Cooperative Oncology Group. Am J Clin Oncol 1982; 5: 649-655.

12 Colinet B, Jacot W, Bertrand D, et al. A new simplified comorbidity score as a prognostic factor in non-small-cell lung cancer patients: description and comparison with the Charlson's Index. Br J Cancer 2005; 93: 1098-1105.

13 Ball D, Thursfield V, Irving L, et al. Evaluation of the Simplified Comorbidity Score (Colinet) as a prognostic indicator for patients with lung cancer: a cancer registry study. Lung Cancer 2013; 82: 358-361.

14 Australian Bureau of Statistics. IRSAD. Canberra: ABS, 2014. http://www.abs. gov.au/ausstats/abs@.nsf/Lookup/2 033.0.55.001main+features100042011 (accessed Mar 2015).

15 Ost D, Yeung S, Tanoue L, Gould M. Clinical and organizational factors in the initial evaluation of patients with lung cancer diagnosis and management of lung cancer, 3rd ed: American College of Chest physicians evidence-based clinical practice guidelines. Chest 2013; 143: sl21S-el41S.

16 Geiger GA, Kim MB, Xanthopoulos EP, et al. Stage migration in planning $\mathrm{PET} / \mathrm{CT}$ scans in patients due to receive radiotherapy for non-small-cell lung cancer. Clin Lung Cancer 2014; 15: 79-85.

17 Kishan AU, Cui J, Wang PC, et al. Quantification of gross tumour volume changes between simulation and first day of radiotherapy for patients with locally advanced malignancies of the lung and head/neck. J Med Imaging Radiat Oncol 2014; 58: 618-624.

18 Conron M, Phuah S, Steinfort D, et al. Analysis of multidisciplinary lung cancer practice. Intern Med J 2007; 37: $18-25$.

19 Burmeister $\mathrm{BH}$, Zarate DD, Burmeister $E A$, et al. Lung cancer patients in Queensland suffer delays in receiving radiation therapy - but not as a result of distance. Intern Med J 2010; 40: 126-132.
20 Ouwens MM, Hermens RR, Termeer RA, et al. Quality of integrated care for patients with nonsmall cell lung cancer: variations and determinants of care. Cancer 2007; 110: 1782-1790.

21 Jakobsen E, Green A, Oesterlind K, et al. Nationwide quality improvement in lung cancer care: the role of the Danish Lung Cancer Group and Registry. J Thor Oncol 2013; 8: 1238-1247.

22 NHS England. Everyone counts: planning for patients 2014/15 to 2018/ 19. Leeds: NHS England, 2013. https:// www.england.nhs.uk/wp-content/ uploads/2013/12/5yr-strat-plann-guidwa.pdf (accessed Nov 2015).

23 Slade C, Talbot R. Sustainability of cancer waiting times: the need to focus on pathways relevant to the cancer type. J R Soc Med 2007; 100: 309-313.

24 Paul C, Carey M, Anderson A, et al. Cancer patients' concerns regarding access to cancer care: perceived impact of waiting times along the diagnosis and treatment journey. Eur $J$ Cancer Care 2012; 21: 321-329.

25 Neal RD, Allgar VL, Ali N, et al. Stage, survival and delays in lung, colorectal, prostate and ovarian cancer: comparison between diagnostic routes. Br J Gen Pract 2007; 57: 212-219.

26 Bergamo C, Lin JJ, Smith C, et al. Evaluating beliefs associated with late-stage lung cancer presentation in minorities. J Thor Oncol 2013; 8 : 12-18.

27 Victorian Cancer Registry. Victorian cancer statistics: regional statistics [website]. http://vcrdata.cancervic.org. au:8082/ccv/\#regional (accessed Nov 2015).

28 Dunican $\mathrm{E}$, Uzbeck M, Clince J, et al. Outcomes of patients presenting to a dedicated rapid access lung cancer clinic. Ir Med J 2011; 104: 265-268.

29 Murray PV, O'Brien ME, Sayer R, et al. The pathway study: results of a pilot feasibility study in patients suspected of having lung carcinoma investigated in a conventional chest clinic setting compared with a centralised two-stop pathway. Lung Cancer 2003; 42: 283-290. 